\title{
Effects of special exercise programs on functional movement screen scores and injury prevention in preprofessional young football players
}

\author{
Engin Dinc ${ }^{1}$, Bekir Eray Kilinc ${ }^{2 *}$, Muge Bulat ${ }^{3}$, Yunus Turgay Erten ${ }^{4}$, Bülent Bayraktar ${ }^{3}$ \\ ${ }^{1}$ Konya Training and Research Hospital, Konya, Turkey \\ ${ }^{2}$ Golhisar State Hospital, Burdur, Turkey \\ ${ }^{3}$ Istanbul University, Istanbul, Turkey \\ ${ }^{4}$ Bulent Ecevit University, Zonguldak, Turkey
}

To increase movement capacity and to reduce injury risk in young soccer players by implementing a special functional exercise program based on functional movement screen (FMS) and correctives. 67 young male athletes 14-19 years of age from a Super League Football Club Academy participated in the study. Functional movement patterns were evaluated with FMS assessment protocol. Deep squat, hurdle step, inline lunge, shoulder mobility, active straight leg raise, trunk stability push-up, and rotatory stability were examined in FMS. Considering the FMS scores the number of intervention and control groups were defined as 24 and 43, respectively. Intervention program was composed of $1 \mathrm{hr}$ twice a week sessions in total of 12 weeks with 4 weeks of mobility, 4 weeks of stability, and 4 weeks of integration exercises. At the end of 12-week intervention and control groups were re-evaluated with FMS protocol. Contact and noncontact sports injuries recorded during one season. In intervention group there was statistically significant difference in increase in total FMS scores $(P<0.01)$, deep squat $(P \leq 0.001)$, hurdle step $(P<0.05)$, inline lunge $(P<0.01)$, and trunk stability push-up $(P<0.01)$. In control group total FMS, deep squat, and trunk stability push-up scores increased with a statistical difference $(P<0.01, P<0.05$, $P \leq 0.01$, respectively). The incidence of noncontact injury in control group was higher than intervention group $(P<0.05)$. Periodic movement screening and proper corrections with functional training is valuable in order to create better movement capacity to build better physical performance and more effective injury prevention.

Keywords: Functional movement screen, Injury prevention, Movement capacity, Physical performance, Preparticipation screening

\section{INTRODUCTION}

At the athletic performance and injury prevention centers, during the last 20 years, sports medicine has evolved into a process involving proprioceptive neuromuscular stimulation, muscle synergy and motor learning skills far more than traditional, isolated force ratings. The functional behavior patterns of the athletes are of great importance for excellent and comprehensive rehabilitation, such as the core strengths are at a high level, the risk of injury is reduced (Cook et al., 2006a; Huxel Bliven and Anderson, 2013). Where there is no functional evaluation standard, it is very difficult to develop a motion approach that we can call functional, creating a gold standard screening test that evaluates functional motion capacities (Garrison et al., 2015).

On the improper motion patterns (mobilization, stabilization, and constraint in motor controls) of the athletes, in order to perform high level activities, unaware that they should do more strength training adversely affect the development of athletes and increases the risk of injury (Bardenett et al., 2015; Cook et al., 2006b).

At the end of the rehabilitation process, specific tests are applied to the performance and sports branch to determine whether the athlete is competent for sportive activity and return to the
${ }^{*}$ Corresponding author: Bekir Eray Kilinc (D) https://orcid.org/0000-0003-1229-9815 Golhisar State Hospital, Fatih Mahallesi Golhisar Devlet Hastanesi 15300 Burdur, Turkey

Tel +90-2484112070, Fax +90-5306061884, E-mail: dreraykilinc@gmail.com Received: August 3, 2017 / Accepted: October 12, 2017
This is an Open Access article distributed under the terms of the Creative Commons Attribution Non-Commercial License (http://creativecommons.org/licenses/by-nc/4.0/) which permits unrestricted non-commercial use, distribution, and reproduction in any medium, provided the original work is properly cited. 
field. It is difficult to say that this process gives enough basic information to assess whether the individual is ready to participate in sporting activities (Cook et al., 2006b). Past researchers' perception was that there was no standard assessment system that could assess the physical adequacy of the individual to participate in activities (American Academy of Family Physicians, 2005; Fields and Delaney, 1990).

In accordance with the evaluation results of the functional movement patterns, candidate sportsmen who volunteer in our work, we performed an individual exercise plan and with the implementation of this planning and we intended to reveal the effects on the total functional movement screen (FMS) scores and the incidence, severity, healing process, and loss of work power of sports injuries at a certain time.

This study, in football discipline, was conducted to investigate the effects of specific exercise planning, movement patterns, and injury severities on the athletes who are professionally trained and their turnaround times. To increase movement capacity and to reduce injury risk in young soccer players by implementing a special functional exercise program based on FMS screening and correctives.

\section{MATERIALS AND METHODS}

Our work was approved by the Local Ethics Committee of Istanbul University Faculty of Medicine (approval number: 2012/ 1416-1204). All of the athletes participated in this study with their family volition and their own wishes.

Twenty-four male soccer players were included in the working group who struggling in the super league for at least 3 years, from sportsmen in the age group 14-19, at least $8 \mathrm{hr}$ a week training and who have not experienced the musculoskeletal injuries that would prevent participation in matches or team training in the last 1 month or require medical treatment. Mean age was $16.13 \pm 0.387$ years, mean height was $175 \pm 4.16 \mathrm{~cm}$, mean weight was $69.07 \pm 4.55 \mathrm{~kg}$. Fourty-three male soccer players were included to the control group mean age was $16.42 \pm 1.57$ years, mean height was $175.75 \pm 4.44 \mathrm{~cm}$, mean weight was $70.29 \pm$ $4.89 \mathrm{~kg}$ were included (Table 1). Athletes have been identified from those who are closer to being elite athletes planned to invest

Table 1. Demographic features of athletes in the study and control groups

\begin{tabular}{lccc}
\hline Group & Weight $(\mathrm{kg})$ & Length $(\mathrm{cm})$ & Age $(\mathrm{yr})$ \\
\hline Study & $69.07 \pm 4.55$ & $175.00 \pm 4.16$ & $16.13 \pm 0.38$ \\
Control & $70.29 \pm 4.89$ & $175.75 \pm 4.44$ & $16.42 \pm 0.24$ \\
\hline
\end{tabular}

Values are presented as mean \pm standard deviation. in talking with their coaches.

FMS original kits were used as material and consist of main test body, two auxiliary fixed measuring sticks, elastic rope, one moving measuring stick (Fig. 1).

FMS consists of 7 movement tests and 3 of these tests consist of control tests that help us check for pain.

FMS tests: 1. Deep squat; 2. hurdle step; 3. in-line lunge; 4. shoulder mobility; 5 . active straight leg raise; 6 . trunk stability push-up; 7. rotary stability.

Control tests: 1 . Shoulder mobility; 2. trunk stability push-up; 3. rotary stability.

The FMS scoring consisted 4 different options between the range of 0 to 3 and the 3 was the best possible score. If the individual feels pain in any part of his body during the test, the athlete will score 0 if he can not complete the movement pattern or could not bring his body to the position score 1 , if the individual can complete the motion by compensating in any way 2 , if the individual movements were not using any compensation mechanism and were able to exercise within the standard expectations of motion, would get 3 points.

The athletes were divided into three groups for test execution. Each test unit was shown to athlete by the researcher practically. What to do during the test was said verbal and it was confirmed that there is no question about application of the test. It was done 3 times after making sure that the athletes knew about the application of the test.

\section{Exercise program}

Twelve weeks special exercise planning that contains 3-gradual exercise program including respectively mobilization, stabiliza-

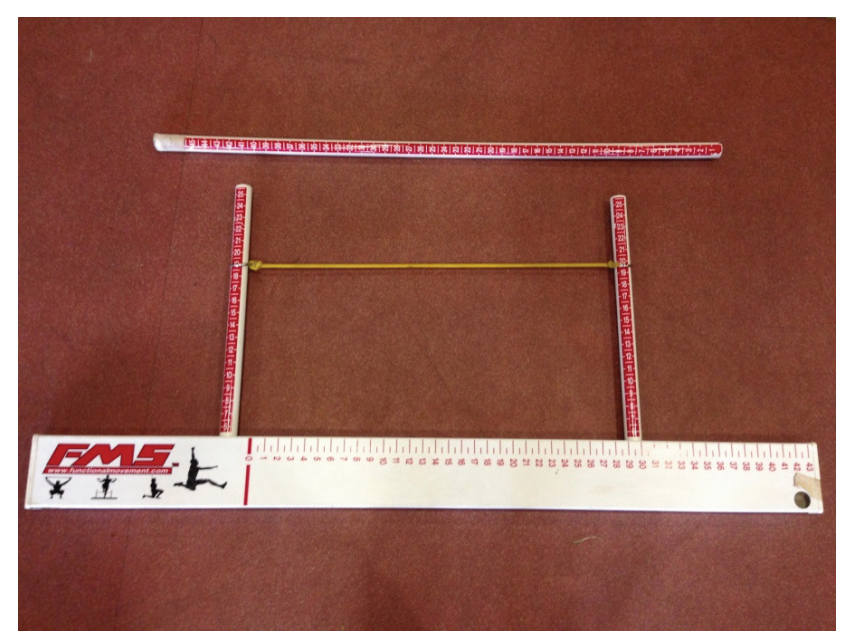

Fig. 1. Test materials. 
tion, and integration was done to the athletes included in the study group. Each program consists of 6-8 session and contains 21-24 session in total. The mobilization exercises were planned to include the upper and lower limb major muscle groups, with foam roller for 3 min each for each muscle group slacken, as well as iliotibial band, posterior capsule of the shoulder joint, thoracic vertebrae, stretching exercises to the posterior capsule of the hip joint and diaphragmatic respiratory exercises. Firstly the exercises were specially determined according to the athletes' test scores and views, then they were applied as 2 days, 1 each hour in addition to team workouts.

In the second 4 weeks, stabilization exercises for the athletes were planned functionally in accordance with the algorithm proposed by Cook according to the athletes' test scores and views, examining the yoga-pilates movements in order to activate the central muscles and examining the deficiencies found in the deep squat, in-line lunge and rotary stability test specially (Cook, 2010).

In the third 4 weeks exercise planning to improve the motor skills of the athletes was performed using resistant rubber, $65-\mathrm{cm}$ pilates ball in accordance with the algorithms and functional motion patterns proposed by Cook in particular, according to the test scores and images of the athletes, in order to get rid of the deficiencies in the techniques of the sports integration exercises deep squat, in-line lunge, trunk stability push-up, and other test steps.

FMS tests of the athletes who completed 18-21 sessions in total applied again and recorded by video. Immediately afterwards, test scores were checked by the same researcher with video recordings and the total FMS scores were determined.

Injury that taken place in contact or noncontact form throughout the season from the start of the working process of the athletes, in the course of training and during the match; that requires medical or physical therapy from club health department; and that hinder training and taking the field were recorded during a session. In all injuries, the diagnosis was recorded by the investigator, according to the scales on which the severity of Union of European Football Associations injuries was determined. Injury that left the athlete less than 7 days on the training or competition was recorded as grade 1 injury; grade 2 injury was recorded as injuries, which left the team training or competition from 7 to 21 days apart, injuries that left the team separate from training or competition during 21 days were recorded as grade 3 injuries.

\section{Statistical analysis}

IBM SPSS Statistics ver. 21.0 (IBM Co., Armonk, NY, USA) was used for data analysis of our study. $P<0.05$ values were ac- cepted statistically significant in all analyzes.

The total FMS, deep squat, hurdle step, in-line lunge, shoulder mobility, active straight leg raise, trunk stability push-up, rotary stability grades before and after the exercise program of whom prospective athletes were compared in the study and control groups and paired sample $t$-test for analysis. The independent sample test was applied to analyze the type and severity of injuries of athletes that saved during a season.

\section{RESULTS}

The average total FMS score of study group, before specific exercise planning was $14.83 \pm 1.46$ and after the study it was $16.79 \pm 1.61$. In the control group, after first measurements the average total FMS scores was $14.95 \pm 1.19$ and after second measurements it was $15.33 \pm 1.19$ (Table 2). These changes were statistically significant both in the study group $(P<0.001)$ and in the control group $(P<0.001)$.

\section{Results of FMS and injury relationship}

The number of days of the study and control group was away from work with the average team during a season due to musculoskeletal injuries were $14.08 \pm 13.5$ and $29.42 \pm 30.67$, respectively (Table 3).

Players of the study and the control groups received the average minutes of official and preparatory competitions during a season were 1,496.72 \pm 846.21 and 1,273.67 \pm 922.72 (Table 3).

The average day spent away from team training or match was $14.08 \pm 13.5$ in the study group, while this duration was $29.42 \pm$ 30.67 in the control group. And this difference was found statistically significant in the study group $(P<0.01)$ (Table 3$)$.

Table 2. Change of total functional movement screen (FMS) scores in the study and control groups

\begin{tabular}{lccc}
\hline Group & Previous FMS & Next FMS & $P$-value \\
\hline Study & $14.83 \pm 1.46$ & $16.79 \pm 1.61$ & $<0.001$ \\
Control & $14.95 \pm 1.19$ & $15.33 \pm 1.19$ & $<0.001$ \\
\hline
\end{tabular}

Values are presented as mean \pm standard deviation.

Table 3. Duration of return to the field of study and control groups

\begin{tabular}{lccc}
\hline Group & $\begin{array}{c}\text { Stay away from } \\
\text { the team }\end{array}$ & Duration $(\mathrm{min})$ & $P$-value \\
\hline Study & $14.08 \pm 13.50$ & $1,496.72 \pm 846.21$ & 0.006 \\
Control & $29.42 \pm 30.67$ & $1,273.67 \pm 922.72$ & 0.333 \\
\hline
\end{tabular}

Values are presented as mean \pm standard deviation. 
Table 4. Injury severity in the study and control groups

\begin{tabular}{lccc}
\hline Grade & Study group & Control group & $P$-value \\
\hline 1 injury (<7 days) & $0.92 \pm 0.77$ & $0.91 \pm 1.08$ & 0.96 \\
2 injury (7-28 days) & $0.50 \pm 0.65$ & $0.74 \pm 0.81$ & 0.21 \\
3 injury (>28 days) & $0 \pm 0$ & $0.24 \pm 0.43$ & 0.001 \\
\hline
\end{tabular}

Values are presented as mean \pm standard deviation.

\section{FMS and injury severity relationship}

Grades 1 and 2 injuries rates were similar in the study and control group athletes and the difference was statistically insignificant $(P=0.96, P=0.21)$. Grade 3 injuries were seen in this level in 10 sportsmen in control group, there was no athlete exposed to injury in the study group. The difference between the two groups was found statistically significant $(P=0.001)$ (Table 4).

\section{FMS and injury mechanism relationship}

The average of contact injuries' values found similar in the study and control groups. There was no statistically difference $(P=0.97)$. The average of noncontact injuries was significantly higher the control group than the study group $(P=0.04)$ (Table 5).

\section{DISCUSSION}

FMS began to be used increasingly to determine the movement patterns of sportsmen at risk of injury in professional clubs. FMS is being used increasingly in professional clubs to determine the patterns of motion of athletes bearing the risk of injury (Chorba et al., 2010). In addition to FMS is a test model designed to assess these weaknesses, limitations, and quality of functional movement patterns, scientific studies have also shown that there is a significant relationship between FMS scores and sport injuries (Cook et al., 2006a; Cook, 2010; Kiesel et al., 2007; Kiesel et al., 2008).

According to the hypothesis suggested that inappropriate motion patterns make individuals susceptible to recurrent musculoskeletal injuries that caused by micro traumas (Cook, 2010). These repetitive micro traumas cause acute injuries that accumulate in the long term and leave the athletes away from the team (Cook et al., 2006b). When the FMS score is 14 or less in the athletes followed during the season, it has been shown that the risk of lower extremity injuries is increased four times (Bardenett et al., 2015; Song et al., 2014).

A study assessed the relationship between FMS scores and the incidence of injuries on 46 professional footballers and found that they had an 11 fold higher risk of injury than the FMS score of 14 or less and $51 \%$ had experienced at least one serious injury during
Table 5. Functional movement screen and injury types

\begin{tabular}{lccc} 
& Study group & Control group & $P$-value \\
\hline Contact Injuries & $0.67 \pm 0.70$ & $0.67 \pm 0.86$ & 0.97 \\
Noncontact injuries & $0.75 \pm 0.67$ & $1.21 \pm 1.12$ & 0.04 \\
\hline
\end{tabular}

the season (Kiesel et al., 2007).

FMS tests fulfills the gap between preaccession testing and performance testing by providing an assessment of individual dynamic and functional movement capacities. Once this test system has completed the rehabilitation process of the athlete and after surgery, as well as helping to make the decision to return to the spor, allowing for different approaches to prevention of injuries and predictability of performance (Cook et al., 2006a; Kiesel et al., 2007).

Nowadays researchers have revealed that individuals who don't have adequate FMS scores or have dysfunctional movement patterns can develop their dysfunctional movement patterns and FMS scores with corrective exercise (Cook et al., 2006b; Letafatkar et al., 2014). The purpose of corrective exercises is to solve measurable dysfunctional movement patterns (Cook, 2010).

A study reported that an 8-week individual exercise program for 25 professional martial arts sportsmen during training sessions, 4 days a week in accordance with the exercise programs recommended by Cook, and found that the total FMS scores increased at the end of the program (Bodden et al., 2015). In our study, we made use of downward facing dog, breathing techniques, warrior poses, balance exercise from yoga exercise while we were preparing exercise programs, in addition to exercise planning developed by Cook. In our study group, we put forward that the average of total FMS scores increased at the end of exercise program. Findings in our study were found to support Bodden and Cowen's work on the contrary researches that claims that specific exercise programs have no positive effect on movement patterns. Based on the results of these studies we can say that the contribution to the development of movement patterns of exercise programs targeting the limitation of movement patterns is more effective than exercise programs targeting performance enhancement with body control and coordination (Bodden et al., 2015; Frost et al., 2012; Kiesel et al., 2011).

Some investigations have revealed that team workouts have a positive effect on the FMS scores of athletes even when specific exercise programs are not being applied in addition to regular team training (Kiesel et al., 2011; Sprague et al., 2014). In our study, we found statistically significant increases in the total FMS scores, Deep squat and trunk stability push-up tests of the athletes in the control group who regularly trained the team and did not partici- 
pate in the specific exercise program. In our study, the athletes in the control group, as a reason for the significant development of both total FMS scores and deep squat and trunk stability push-up, has been thought that the presence of the core muscle group stabilization, balance and coordination exercises in the general training approach is considered to be effective and as the other reason that a number of them may be influenced by the determination of treatment programs through exercise programs that will affect functional movement patterns depending on the sporting injuries they have had in the process. In the active straight leg raise test, it is considered that the reason for the lack of regression is that stretching exercises appropriate to the Fédération Internationale de Football Association 11+ exercise program are made before and after each training.

Nowadays, speed and importance are given to the studies about the protection from injuries and the determination of the causes of injuries and the reduction of their risks. Some of these studies are trying to establish the necessary predictions in terms of preventive medicine in sports injuries by evaluating the relationship between FMS scores and injuries.

In the study they made in basketballer pointed out that FMS scores were associated with noncontact injuries and that there was no relation between contact injuries and functional movement capacity, but those who had good functional movement were able to avoid contact injuries (Sorenson, 2009). Also in our study, the number of non-contact injuries was higher in the control group than in the study group, and the difference was determined statistically significant. We could not reveal a statistically significant difference between contact injuries and total FMS scores. Assessed the total FMS scores of the 82 basketballer high school students before the season and after the season, at the same time they recorded the musculoskeletal injuries causing athletes to stay away from the team during a season, they were not found a statistically significant difference between the total FMS scores of the athletes who were exposed to injury at the end of the season and the total FMS scores of those who did not. We also not found a statistically significant difference between the total FMS scores and the total number of injuries in our study.

A study showed that the musculoskeletal injuries which require medical treatment and physiotherapy and the rate of injury to the athletes with a total FMS score lower than 14, showed that there was a significant relationship between the total FMS score and the risk of injury when those who had previously suffered anterior cruciate ligament (ACL) injury in the study were out of the group (Chorba et al., 2010). While 11 of the 15 athletes (73.3\%) who were not injured in the ACL in the past and whose FMS score was lower than 14 were injured; 6 of the 16 athletes were injured in the athletes group with FMS score higher than 14. A study showed that the definition of serious injury as musculoskeletal injuries that requiring treatment for more than 3 weeks and at the end of study they showed that athletes who have FMS score of 14 or less have an 11 times higher risk of injury and that the athletes are exposed to $51 \%$ serious injury during the season (Garrison et al., 2015). There were no ACL injuries in any athlete participating in our study. While there is not any athlete have total FMS score below 14 in our study group, there is 2 athletes in control group was measured below 14 . Taken place in the study and control group the difference between number of grade 3 injury was found statistically significant. The severity of the injuries of the athletes over 3 weeks, the rate of injuries to the sportsmen in the special exercise program was found $25.8 \%$, and $74.2 \%$ in the sportsmen in the control group.

The core force distributed the energy transfer between the upper and lower extremities reported that may cause an increase in musculoskeletal injuries such as force imbalances due to the effect of contributing to neuromuscular control during functional motion patterns and providing body stabilization, the core strength developed due to the lack of stabilization in undeveloped athletes and compensation movement patterns (Kiesel et al., 2007). There is a statistically significant relationship between the lower extremity injuries in this parameter because of that the trunk stability is an important indicator of central stabilization of the spine (Granacher et al., 2013; Leetun et al., 2004; Lephart et al., 2002; Paterno et al., 2004). However in our study; we could not found a statistically significant relationship between the number of injuries, type and severity, and trunk stability push-up test. It is possible to improve the dysfunctional movement patterns and to increase FMS scores with special exercise programs. When considered the relationship between injuries and total FMS scores, the severity of injuries suffered by the athlete is inversely related to the FMS scores, not with specific exercise planning. In terms of preventive medicine, individual exercise programs based on FMS scores were thought to be beneficial in terms of reducing the number of injuries and reducing the severity of injuries.

Periodic movement screening and proper corrections with functional training is valuable in order to create better movement capacity to build better physical performance and more effective injury prevention. Since FMS are used during sportive activities, to try to understand the common aspects of these movement patterns and to make exercise programs for the development of 
movement patterns will provide considerable contributions to the reduction of job losses and economic losses as provided by athletes to increase their athletic performances, protect themselves from injury, and stay with the team for longer.

\section{CONFLICT OF INTEREST}

No potential conflict of interest relevant to this article was reported.

\section{REFERENCES}

American Academy of Family Physicians. The preparticipation physical evaluation. 3rd ed. New york: Mcgraw-hill; 2005.

Bardenett SM, Micca JJ, DeNoyelles JT, Miller SD, Jenk DT, Brooks GS. Functional movement screen normatıve values and valıdity in high school athletes: can the FMS ${ }^{\mathrm{TM}}$ be used as a predictor of injury? Int J Sports Phys Ther 2015;10:303-308.

Bodden JG, Needham RA, Chockalingam N. The effect of an intervention program on functional movement screen test scores in mixed martial arts athletes. J Strength Cond Res 2015;29:219-225.

Chorba RS, Chorba DJ, Bouillon LE, Overmyer CA, Landis JA. Use of a functional movement screening tool to determine injury risk in female collegiate athletes. N Am J Sports Phys Ther 2010;5:47-54.

Cook G. Movement: functional movement systems: Screening, assessment, corrective strategies. Santa Cruz (CA): On Target Publications; 2010.

Cook G, Burton L, Hoogenboom B. Pre-participation screening: the use of fundamental movements as an assessment of function - part 1. N Am J Sports Phys Ther 2006;1:62-72.

Cook G, Burton L, Hoogenboom B. Pre-participation screening: the use of fundamental movements as an assessment of function - part 2. N Am J Sports Phys Ther 2006;1:132-139.

Fields KB, Delaney M. Focusing the preparticipation sports examination. J Fam Pract 1990;30:304-312.

Frost DM, Beach TA, Callaghan JP, McGill SM. Using the Functional Movement Screen ${ }^{\mathrm{TM}}$ to evaluate the effectiveness of training. J Strength Cond Res 2012;26:1620-1630.

Garrison M, Westrick R, Johnson MR, Benenson J. Association between the functional movement screen and injury development in college athletes. Int J Sports Phys Ther 2015;10:21-28.

Granacher U, Gollhofer A, Hortobágyi T, Kressig RW, Muehlbauer T. The importance of trunk muscle strength for balance, functional performance, and fall prevention in seniors: a systematic review. Sports Med 2013;43:627-641.

Huxel Bliven KC, Anderson BE. Core stability training for injury prevention. Sports Health 2013;5:514-522.

Kiesel K, Plisky P, Butler R. Functional movement test scores improve following a standardized off-season intervention program in professional football players. Scand J Med Sci Sports 2011;21:287-292.

Kiesel K, Plisky P, Kersey P. Functional movement test score as a predictor of time-loss during a professional football team's pre-season. Med Sci Sports Exerc 2008;40:S234.

Kiesel K, Plisky PJ, Voight ML. Can serious injury in professional football be predicted by a preseason functional movement screen? N Am J Sports Phys Ther 2007;2:147-158.

Leetun DT, Ireland ML, Willson JD, Ballantyne BT, Davis IM. Core stability measures as risk factors for lower extremity injury in athletes. Med Sci Sports Exerc 2004;36:926-934

Lephart SM, Ferris CM, Riemann BL, Myers JB, Fu FH. Gender differences in strength and lower extremity kinematics during landing. Clin Orthop Relat Res 2002;(401):162-169.

Letafatkar A, Hadadnezhad M, Shojaedin S, Mohamadi E. Relationship between functional movement screening score and history of injury. Int J Sports Phys Ther 2014;9:21-27.

Paterno MV, Myer GD, Ford KR, Hewett TE. Neuromuscular training improves single-limb stability in young female athletes. J Orthop Sports Phys Ther 2004;34:305-316.

Song HS, Woo SS, So WY, Kim KJ, Lee J, Kim JY. Effects of 16-week functional movement screen training program on strength and flexibility of elite high school baseball players. J Exerc Rehabil 2014;10:124-130.

Sorenson EA. Functional movement screen as a predictor of injury in high school basketball athletes [dissertation]. Eugene (OR): University of Oregon; 2009.

Sprague PA, Mokha GM, Gatens DR. Changes in functional movement screen scores over a season in collegiate soccer and volleyball athletes. J Strength Cond Res 2014;28:3155-3163. 